\title{
Superconducting atom chips: advantages and challenges
}

\author{
V. Dikovsky ${ }^{1}$, V. Sokolovsky ${ }^{2}$, Bo Zhang ${ }^{3}$, C. Henkel ${ }^{3}$, and R. Folman ${ }^{1,2}$ \\ ${ }^{1}$ Ilse Katz Center for Meso- and Nanoscale Science and Technology, \\ Ben-Gurion University of the Negev, Beer Sheva, Israel \\ ${ }^{2}$ Department of Physics, Ben-Gurion University of the Negev, Beer Sheva, Israel \\ ${ }^{3}$ Institut für Physik und Astronomie, Universität Potsdam, Potsdam, Germany
}

21 July 2008

\begin{abstract}
Superconductors are considered in view of applications to atom chip devices. The main features of magnetic traps based on superconducting wires in the Meissner and mixed states are discussed. The former state may mainly be interesting for improved atom optics, while in the latter, cold atoms may provide a probe of superconductor phenomena. The properties of a magnetic side guide based on a single superconducting strip wire placed in an external magnetic field are calculated analytically and numerically. In the mixed state of type II superconductors, inhomogeneous trapped magnetic flux, relaxation processes and noise caused by vortex motion are posing specific challenges for atom trapping.
\end{abstract}

PACS: 37.10.Gh Atom traps and guides 85.25.Am Superconducting device characterization, design, and modeling

\section{Introduction}

One of the high priority goals in atom chip research is to increase lifetime and coherence time for ultracold atoms trapped in magnetic potentials close to the surface. This is important for both scientific aims and technological applications. 
Progress towards this goal demands the control and reduction of magnetic noise produced by the metallic components of the atom chip. Randomly fluctuating magnetic fields are generated by thermal current noise in the conducting chip elements and reduce the number of trapped atoms (losses), increase their temperature (heating) and lead to a phase uncertainty in the atom's state (decoherence) - see, for example, [1, 2, 3] and references therein. Theoretical analysis of the magnetic noise generated by a normal metal [4, 5, 6, 7] predicts a fast reduction of the lifetime with the decrease of the distance $z_{t}$ between the trapped atom and the metal surface (trap height); this is in excellent agreement with lifetime measurements [8, 9, 10]. At a trap height less than $10 \div 20 \mu \mathrm{m}$, thermal magnetic noise exceeds all other harmful influences on the atom cloud (technical noise due to the current supply instability, residual gas collisions, stray magnetic fields) and provides the dominant limit for the lifetime [1, 3]. In the last few years, the application of superconducting materials to atom chips has been widely discussed as a perspective to extend the lifetime of cold atoms [7, 11, 12, 13, 14]. A recent theoretical estimate [13] of the magnetic noise caused by a superconductor in the Meissner state showed that the lifetime of atoms trapped above a superconducting layer would be, at least, six orders of magnitude longer than above a normal metal in the same geometry. The analysis presented in [14] predicts an atom lifetime of $5000 \mathrm{~s}$ at a trap height of $1 \mu \mathrm{m}$. For comparison, at the same height in a normal metal trap the lifetime is less than $0.1 \mathrm{~s}$ [9]. At larger heights, the lifetime in a superconducting niobium chip can be much larger (up to $10^{11} \mathrm{~s}$ at temperature $T=4.2 \mathrm{~K}$ ).

Two first realizations of atom chips with superconducting elements have been reported in Refs.[15] and [16]. In both setups, the trapped atoms were ${ }^{87} \mathrm{Rb}$. In the experiment by Nirrengarten et al. [15], the current-carrying wires (in " $U$ " and " $Z$ " shape) were made of niobium and operated at about $4.2 \mathrm{~K}$. The obtained atom spin relaxation time was estimated as $115 \mathrm{~s}$. This value is comparable to the best one achieved for atoms trapped near normal-metal wires [17]. In the second experiment [16], special efforts have been undertaken to reduce the influence of technical noise. Using a $\mathrm{MgB}_{2}$ film, a " $\mathrm{Z}$ "-shaped wire was fabricated as a part of a closed superconducting loop and operated in the persistent current regime. This permits to disconnect the current supply and get rid of its instability, i.e. technical noise. To our knowledge, in both experiments the trap lifetimes were limited by processes other than the magnetic noise generated by the superconducting elements of the atom chip.

Aside from magnetic noise reduction (thermal and technical), the application of superconductors in atom chips may be advantageous due to high current den- 
sities without Joule heating, and practically zero electric fields across the superconducting elements. Atom traps with high currents and strong confinement, as needed for some applications, may not be operated in the Meissner state because of too low critical parameters. One can then use superconducting wires in the so-called mixed state where magnetic flux partially penetrates the chip structures. This is an issue that we address in this paper, complementing previous approaches that focus on the Meissner state [20]. It is well known that in addition to atom optics, one may use cold atoms as sensitive probes of current distribution and noise in the nearby surface of the atom chip [18, 19]. As the mixed state of a superconductor exhibits vortex phenomena and flux noise much higher than that of the Meissner state, it may be an interesting object to study in this context, beyond the advantages of carrying larger super currents. The results reported here identify a fairly large parameter window where atom chips can be designed with superconducting elements, both in the Meissner and mixed states.

In the following Section, we review typical properties of superconducting materials, in particular critical parameters in view of atom chip applications. Section 3 analyses side guide traps formed by combining a bias field with the supercurrent of a rectangular wire. We show analytically and numerically how the current distribution is significantly modified due to screening and flux penetration and identify the consequences for trapping and transporting atoms on a chip. We also give an overview on magnetic noise in mixed-state superconductors. Section 4 discusses the confinement of the magnetic trap, compared to normal metal wires. The analytically solvable case of a cylindrical wire and the method used for numerical calculations are described in the Appendices.

\section{Typical superconductors}

Let us briefly survey the properties of superconductors which are important for building a superconducting atom chip. At low enough fields and temperatures, superconductors exhibit the Meissner effect: magnetic fields are expelled from their interior. In this regime, the field penetrates into a superconductor only over a small depth $\lambda$ from the surface - the London penetration depth. At $T=0, \lambda$ is of the order of $50 \mathrm{~nm}$ and increases with temperature as $\left[1-\left(T / T_{c}\right)^{4}\right]^{-1 / 2}$, where $T_{c}$ is the critical temperature.

Superconducting materials are classified as type I and type II superconductors that differ in their behavior as the magnetic field is increased. Type I supercon- 
ductors (pure metals as $\mathrm{Pb}, \mathrm{Al}, \mathrm{Hg}, \mathrm{In}$ ) are in the Meissner state over the whole sub-critical ranges of temperature $T<T_{c}$, external magnetic field $B_{0}<B_{c}$, and current $I<I_{c}$. Their critical parameters are quite low, however, which is the main problem for applications. The highest values are observed for lead: $T_{c}=7.2 \mathrm{~K}$, critical magnetic field $B_{c}=0.055 \mathrm{~T}$ at $T=0 \mathrm{~K}$, and surface (sheet) critical current density $J_{c}=B_{c} / \mu_{0}=4.4 \cdot 10^{4} \mathrm{~A} / \mathrm{m}$ in zero external magnetic field at $T=0$ [21]. ( $\mu_{0}$ is the free space permeability.)

Table 1: Critical parameters of selected type-II superconductors. The critical current density values are referring to the highest quality superconducting films and tapes $\left(\mathrm{Nb}_{3} \mathrm{Sn}\right.$ and $\left.(\mathrm{Pb}, \mathrm{Bi})_{2} \mathrm{Sr}_{2} \mathrm{Ca}_{2} \mathrm{Cu}_{3} \mathrm{O}_{10}\right)$. Below the first critical field $B_{c 1}$, the material is in the Meissner state (vortex free), between $B_{c 1}$ and $B_{c 2}$ in the mixed state (with vortex penetration). The temperature is $4.2 \mathrm{~K}$ unless otherwise quoted. Data collected from Refs. [15, 22, 23, 24, 25, 26, 27, 28, 29, 30].

\begin{tabular}{|l|c|c|c|c|}
\hline Superconductor & $T_{c}(\mathrm{~K})$ & $B_{c 1}(\mathrm{mT})$ & $B_{c 2}(\mathrm{~T})$ & $j_{c}\left(\mathrm{~A} / \mathrm{m}^{2}\right)$ \\
\hline \hline $\mathrm{Nb}$ & 9.3 & 140 & 0.28 & $5 \cdot 10^{10}(B=0)$ \\
\hline $\mathrm{Nb}_{3} \mathrm{Sn}$ & 18 & 40 & 27 & $6 \cdot 10^{10}(B=1 \mathrm{~T})$ \\
\hline $\mathrm{MgB}_{2}$ & 39 & 30 & 15 & $3.5 \cdot 10^{11}(B=0)$ \\
\hline $\begin{array}{l}\mathrm{YBa} \mathrm{Cu}_{3} \mathrm{O}_{7-\delta} \\
(\mathrm{YBCO})\end{array}$ & 92 & $\begin{array}{c}25(B \| a b, T \rightarrow 0) \\
90(B \| c, T \rightarrow 0)\end{array}$ & $>100(77 \mathrm{~K})$ & $7.2 \cdot 10^{11}(B=0)$ \\
\hline$(\mathrm{Pb}-\mathrm{Bi})_{2} \mathrm{Sr}_{2} \mathrm{Ca}_{2} \mathrm{Cu}_{3} \mathrm{O}_{10}$ & 108 & $13(B \| a b, T \rightarrow 0)$ & $>100(77 \mathrm{~K})$ & $\approx 10^{10}(B=0)$ \\
\hline
\end{tabular}

Much higher critical parameters are observed in type II superconductors. We give typical examples of this type in Table 11 including niobium, its compound $\mathrm{Nb}_{3} \mathrm{Sn}$, as well as the high-temperature superconductors (HTSC) $\mathrm{YBa}_{2} \mathrm{Cu}_{3} \mathrm{O}_{7-\delta}$ and $(\mathrm{Pb}-\mathrm{Bi})_{2} \mathrm{Ca}_{2} \mathrm{Sr}_{2} \mathrm{Cu}_{3} \mathrm{O}_{10}$. These materials exhibit the Meissner effect below the lower critical field, $B<B_{c 1}$, which is relatively small $(50 \div 500 \mathrm{G}$ at $0 \mathrm{~K}$ ). The largest value at $T=0$ is found for niobium $\left(B_{c 1}(0) \approx 170 \mathrm{mT}\right)$ [22]. With increasing temperature, the lower critical field falls down approximately as $B_{c 1}(0)\left[1-\left(T / T_{c}\right)^{2}\right]$, and other parameters $\left(B_{c 2}, j_{c}\right)$ also decrease towards zero as $T \rightarrow T_{c}$. The details of these laws depend on the type of superconductor and its fabrication [21]. In Table 1] we present main critical parameters at $T=4.2 \mathrm{~K}$. For highly anisotropic HTSC with layered crystal structures, the lower critical field (as well as the critical current density) depends on the direction of the field relative to the $a b$-planes [31]. In magnetic fields (external or caused by a transport current) higher than $B_{c 1}$, the magnetic flux penetrates into a type II superconductor in the form of vortices that arrange into a more or less regular flux-line lattice 
(Abrikosov lattice) which is pinned by inhomogeneities of the material [21]. Each vortex carries one quantum of magnetic flux $\Phi_{0}=\pi \hbar / e=2.07 \cdot 10^{-15} \mathrm{~T} \mathrm{~m}^{2}(e$ is the electron charge and $\hbar$ the Planck constant). In the simplest (isotropic) case, it looks like a cylindrical tube of radius $\sim \lambda$ in which superconducting screening currents circulate around a normally conducting core of radius $\sim \xi$ (superconductor coherence length). Both $\lambda$ and $\xi$ depend on temperature, with $\lambda \gg \xi$ for most type II superconductors. The mixed state is observed up to the upper critical field $B_{c 2}$ where the vortex cores merge and the superconducting state is destroyed.

If a superconducting material is used in atom chips, it is particularly important that the lower critical field $B_{c 1}$ and the critical current density $j_{c}$ be large. The second critical field $B_{c 2}$ for most type II superconductors is typically too large to be a relevant limit in the magnetic fields applied in usual atom chip setups. Both lower critical field and critical current density are "technology dependent" because they are very sensitive to crystal defects (for details see Refs. [29, 32, 33]). The $j_{c}$ values collected in Table 1 refer to films and tapes of the best quality. For example, in the atom chip experiments of Refs. [15, 16], the critical current density was $5 \cdot 10^{10} \mathrm{~A} / \mathrm{m}^{2}$ (niobium film) and $10^{11} \mathrm{~A} / \mathrm{m}^{2}\left(\mathrm{MgB}_{2}\right.$ film) respectively.

One specific property, which distinguishes type II superconductors from both normal metals and superconductors in the Meissner state, is their capability to freeze a magnetic flux [21]. This effect is due to pinning forces. The vortices can move under the action of the transport current, when the Lorentz force $j \Phi_{0}$ is stronger than the pinning force, which can be estimated as $j_{c} \Phi_{0}$ where $j_{c}$ is the critical current density. This flux motion results in energy dissipation and induces a voltage drop along the superconducting element. A voltage drop of $1 \mu \mathrm{V} / \mathrm{cm}$ is usually taken as a criterion to define the critical current. Another mechanism of energy dissipation predicted by P. Anderson operates at sub-critical currents and is connected with thermally activated jumps of vortices out of pinning centres, which also generate electric fields [34]. The motion of vortices under various conditions has been investigated by many authors, see for example Ref.[32, 33].

\section{Side guide traps with superconducting wires}

\subsection{Rectangular wire in the Meissner state}

We consider magnetic traps in the "side guide" configuration to illustrate the differences between normal metal and superconducting chips. In this section we analyse a trap based on wires having the form of a thin strip which is the usual 

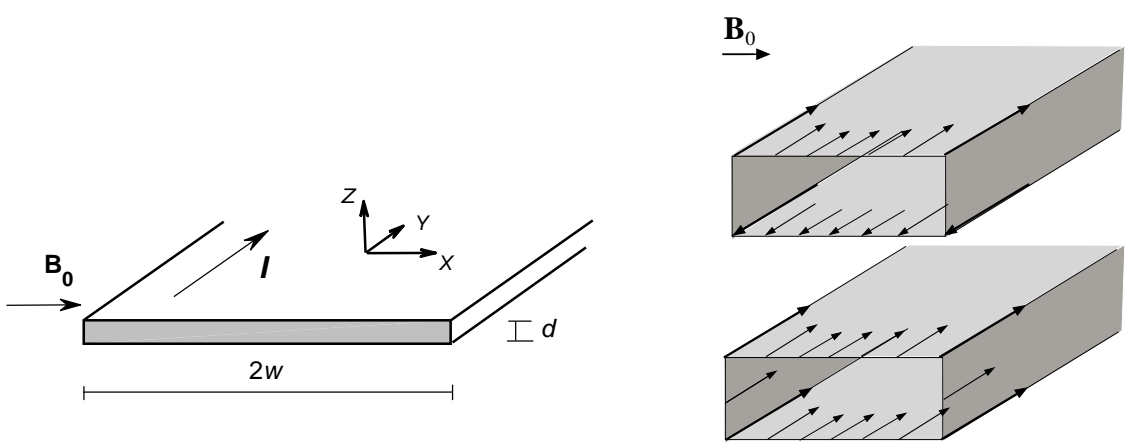

Figure 1: (left) Superconducting strip carrying a current and placed in an external magnetic field. $z=0$ is at the top surface of the strip. (right) Illustration of the surface current distribution (arrows) for the strip wire. Top: screening supercurrents in an external field $\mathbf{B}_{0}$, parallel to the top wire surface (this direction is called "horizontal"). The screening currents are particularly large at the corners, but have opposite directions on the top and bottom faces; the total current is zero. Bottom: transport current without an external field. The current density is maximal near the corners [see also Eq.(1)].

shape in present day atom chips. The case of cylindrical conductors is considered in Appendix $\mathrm{A}$.

\subsubsection{Horizontal bias field}

Let us start with a superconducting strip wire in the Meissner state. The strip is infinitely long along the $y$-axis, with a width $2 w$ along $x$ and a thickness $d$ along $z$, perpendicular to the chip surface (Fig.1). The strip is placed in an external bias field $\mathbf{B}_{0}$ parallel to the $x$-axis. If we assume that the strip is thin, $d \ll w$, the real current density distribution can be replaced by a sheet current $J(x)$ (in $\mathrm{A} / \mathrm{m}$ ) determined by integrating the current density over the strip thickness. The conditions under which these assumptions are fulfilled are discussed in [35, 36]. Regarding the magnetic field around a thin superconducting strip, one expects that the $z$-dependence of the current density can be neglected if the thickness is much less than the observation distance, $z \gg d$. The thin strip approximation is therefore expected to be valid for superconducting films with typical thicknesses of a few hundred nanometers and trap heights above several microns. We confirm this expectation by comparing to numerical calculations that take into account a 
finite thickness 1

We calculate first the current distribution in the strip and then the outer magnetic field using the Bio-Savart law. In this section, we focus on strips in the Meissner state where magnetic flux does not penetrate. Any magnetic field perpendicular to the strip surface is completely shielded by appropriate screening currents. If a bias field $\mathbf{B}_{0}$ is applied parallel to the wide strip surface, there are no screening currents in an infinitely thin strip, and the sheet current is given by the transport current alone. This situation is changed when the finite thickness is taken into account.

For an infinitely thin strip, the sheet current profile along the $x$-axis as well as the field distribution around the strip can be calculated analytically. The sheet current density is given by [35, 36]

$$
J(x)=\frac{I / \pi}{\sqrt{w^{2}-x^{2}}}
$$

The magnetic field above the strip is presented in Fig.2(top). We actually plot its modulus $|\mathbf{B}(\mathbf{r})|$ since this provides the magnetic trapping potential in the adiabatic approximation. Due to symmetry, the minimum modulus of the total field occurs on the $z$-axis (above the centre of the strip) where the field caused by the transport current $I$ is parallel to the $x$-axis:

$$
B_{x}(x=0, z)=\frac{\mu_{0} I z}{2 \pi^{2}} \int_{-w}^{w} \frac{\mathrm{d} x^{\prime}}{\sqrt{w^{2}-x^{\prime 2}}\left(x^{\prime 2}+z^{2}\right)}=\frac{\mu_{0} I}{2 \pi \sqrt{w^{2}+z^{2}}}
$$

This can be compared to the field above a normally conducting strip. Here, the current distribution can be taken as uniform, and the Biot-Savart law gives [2]

$$
\text { normal wire: } B_{x}(x=0, z)=\frac{\mu_{0} I}{2 \pi w} \arctan (w / z)
$$

The field profiles, described by (2) and (3) as well as the numerical results for two thickness/width ratios are plotted in Fig.3. By adding a homogeneous bias field along the $x$-axis with value $B_{0}=-B_{x}\left(x=0, z_{t}\right)$, a magnetic quadrupole trap is formed at height $z_{t}$. We see that for a superconducting strip, the required bias field is smaller than for a normal conductor, by a factor $2 / \pi$ at small trap height. This is a result of the different distribution of current density. Both wires behave practically the same above heights $z \geq 2.5 \mathrm{w}$. Similar results are obtained for a cylindrical wire (see Appendix A).

\footnotetext{
${ }^{1}$ These calculations are outlined in Appendix B. For the sake of faster convergence and to avoid singular fields, we have 'rounded' the edges of the wires with a radius of curvature $r \approx w / 32$.
} 

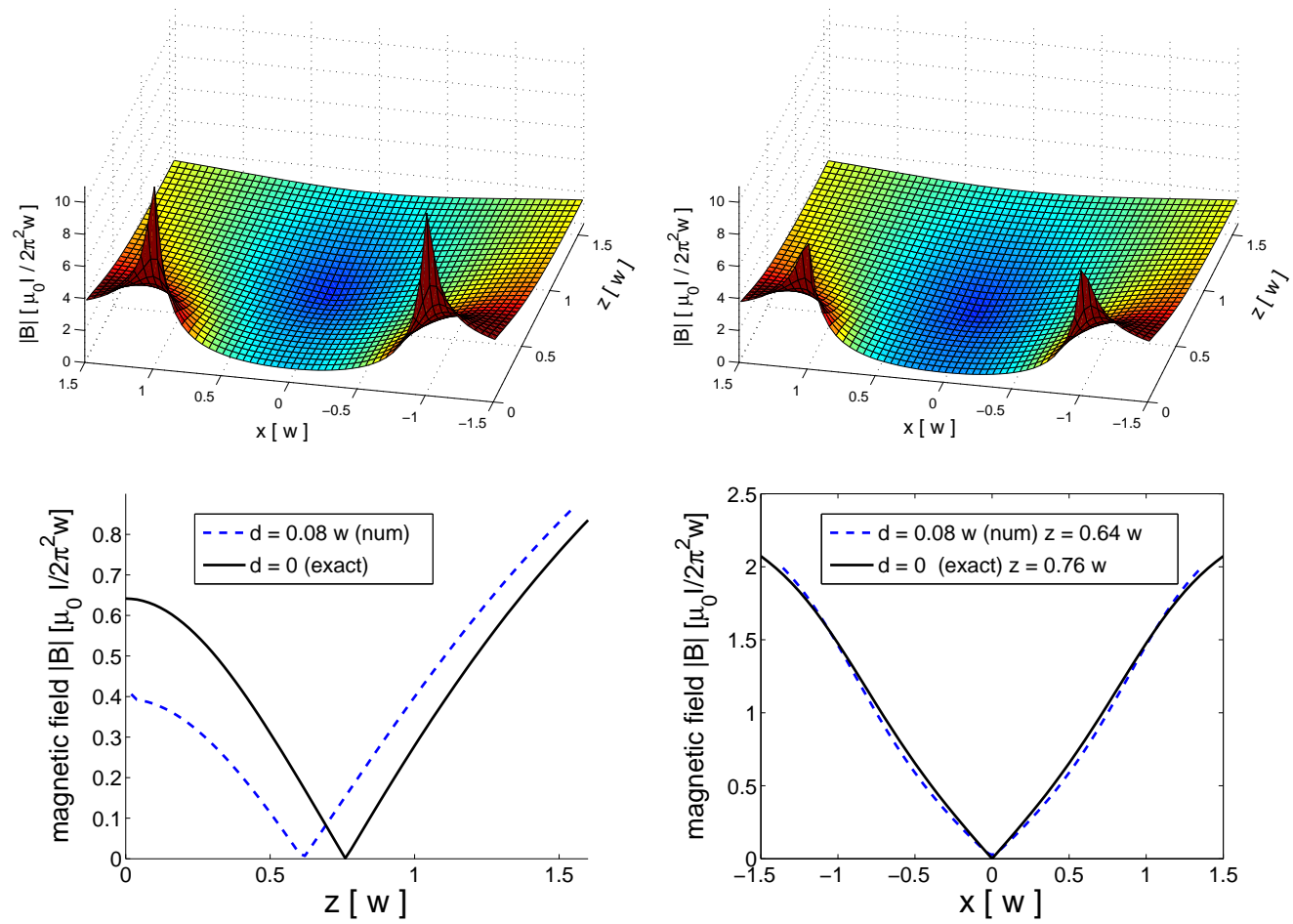

Figure 2: (top) Magnetic trap ("side guide") created by a superconducting rectangular wire with a transport current, placed in a horizontal bias field (as shown in Fig.11). We plot the modulus of the magnetic field in units of $\mu_{0} I /\left(2 \pi^{2} w\right)$. For $I=1 \mathrm{~A}$ and $w=1 \mathrm{~mm}$, this unit corresponds to $(2 / \pi) \mathrm{G}$. (top left) infinitely thin wire (analytical calculation); (top right) finite thickness $d \approx 0.04 \times 2 w$ (numerical calculation). (bottom) Cross-sections along the $z$-axis and $x$-axis through the trap centre ( $x=0$ is chosen in the middle of the top wire face.) Solid lines: analytical results for an infinitely thin strip in the sheet current approximation; dashed lines: numerical calculation for a finite thickness. Due to the finite thickness, the trap is shifted closer to the wire surface (compared to the case $d=0$ the trap height is reduced from $z_{t}=0.76 w$ to $0.64 w$ ). Half of this shift can be explained by measuring the distance from the center of the wire, and shifting the dashed curve by $d / 2$ would make it coincide with the solid curve at large distances. The bias field is $B_{0}=-2.5 \mu_{0} I /\left(2 \pi^{2} w\right)$. The numerical calculations use a thickness $d \approx 0.04 \times 2 w$, with rounded corners (radius $0.031 \mathrm{w}$ ). 

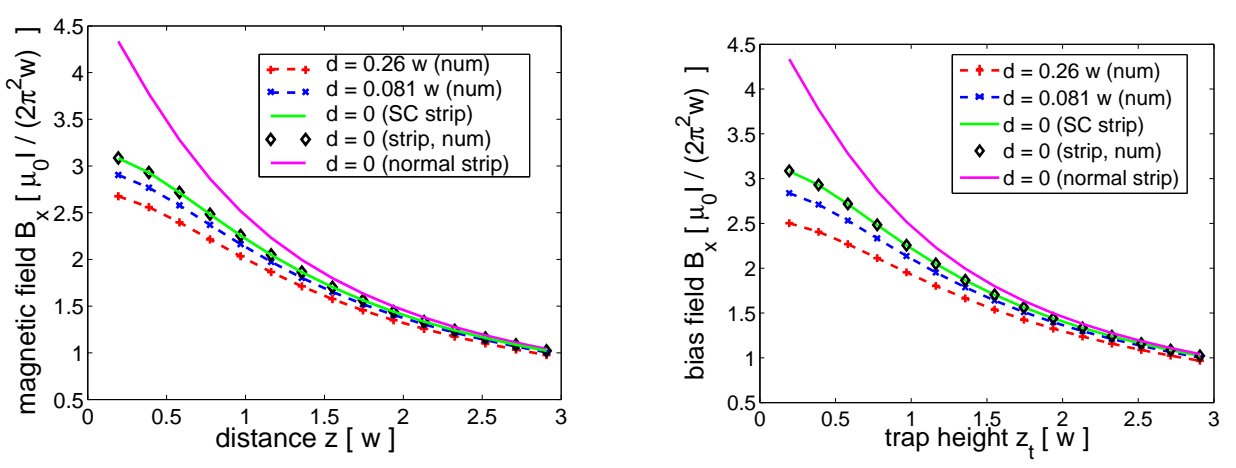

Figure 3: (left) Magnetic field created by a supercurrent $I$ in a rectangular wire as a function of the observation distance $z$ (in units of the strip half-width $w$, measured from the wire top surface). Solid lines give analytical results for $d=0$ : magenta - normal metal strip, green - superconducting strip (superimposed diamonds: numerical calculation). Dashed lines (with symbols) give numerical results for superconducting strip of different thickness: blue $-d=0.04 \times 2 w$, and red $-d=0.13 \times 2 w$. (right) Bias field required to form a trap at distance $z_{t}$ above a strip wire. This plot differs from the left one only for wires of finite thickness where screening currents caused by the bias field are different on the top and bottom surfaces. For this case, the trap height is slightly reduced.

Figs, 2, 3 also illustrate the impact of the finite thickness of the superconducting wire. The bias field induces screening currents on the top and bottom faces of the wire and makes the currents on the top and bottom sides differ (Fig.1 top right). The effect is, however, quite small for the parameters that we explored (up to thickness $d \approx w / 4$ ). For example, the magnetic field gradient in the trap centre is practically unchanged (Fig, 2). The main tendency is to bring the trap position closer to the wire surface (at fixed bias field), see Fig.2. Conversely, the minimum bias field required to create a trap at nonzero height is lowered (Fig 3 , right).

\subsubsection{Vertical bias field}

The influence of a magnetic field on the current distribution in a flat superconducting wire is most pronounced when the field is perpendicular to the wide surface of the wire. For this reason, different loading procedures have to be designed for superconducting atom chips compared to normal ones. Typically, a chip trap is loaded by transporting (positioning) an atom cloud along the $x$-axis adjusting bias fields and currents. In a normal-metal atom chip this is performed using a vertical bias $B_{z}$. Above a superconducting wire, the procedure must be altered as the 
wire builds up significant screening currents to shield its interior from a vertical field. The field profile above the chip becomes significantly non-uniform, leading to potential barriers that have to be taken into account for the cloud transport. This effect is maximal for superconductors in the Meissner state and provides the most striking difference to a normal conductor.

As an example, we illustrate in Fig 4 the magnetic field above a superconducting strip placed in a vertical bias. Magnetic field maxima occur at the edges of the strip (Fig 4(left)) that exceed significantly the applied bias field near the wire surface. This field increase is reduced to about $15 \%$ at a height of $1.5 \mathrm{w}$, but should be taken into account for loading the trap. Note that in order to maintain the Meissner state, the total magnetic field should be less than the lower critical field $B_{c 1}$ in any point of the superconductor surface. The vertical magnetic field near the edges of the superconducting strip increases proportionally to the ratio width/thickness $(2 w / d)$ and may become significant for a wide strip [37]. The same magnetic field increase near the strip edges is induced by the transport current.2 This field concentration can result in the partial transition of the sample into the mixed state even if the current and magnetic fields are far from the critical values. Magnetic traps based on superconducting wires in the mixed states are discussed in the next section.

\subsection{Superconducting guiding wire in the mixed state}

\subsubsection{Side guide}

Let us consider a side guide trap realized by a type II superconducting wire in the form of a strip (Fig.1), carrying a transport current $I$ in zero external field. To calculate the current distribution in the strip we use the Bean critical state model [39]: the current (area) density can only take three different values: $\pm j_{c}$ or 0 . Following Brandt [35], the sheet current (integrated over the thickness of the strip) is determined as $J(x)=\left(d_{+}-d_{-}\right) j_{c}$, where $d_{+}$and $d_{-}$are the history-dependent thicknesses of the regions carrying $+j_{c}$ or $-j_{c}$, respectively $\left(d_{+}+d_{-} \leq d\right)$. The sheet current cannot be higher than its critical value $J_{c}=d j_{c}$. This value is achieved in regions near the strip edges. In the central part of the strip, a field-free region exists that is shielded by the current-carrying domains from the magnetic field, similarly to the Meissner state. In this model for a type II superconducting

\footnotetext{
${ }^{2}$ This is a rough approximation which takes into account only the demagnetisation factor. A more accurate analysis is given, for example, in [38].
} 

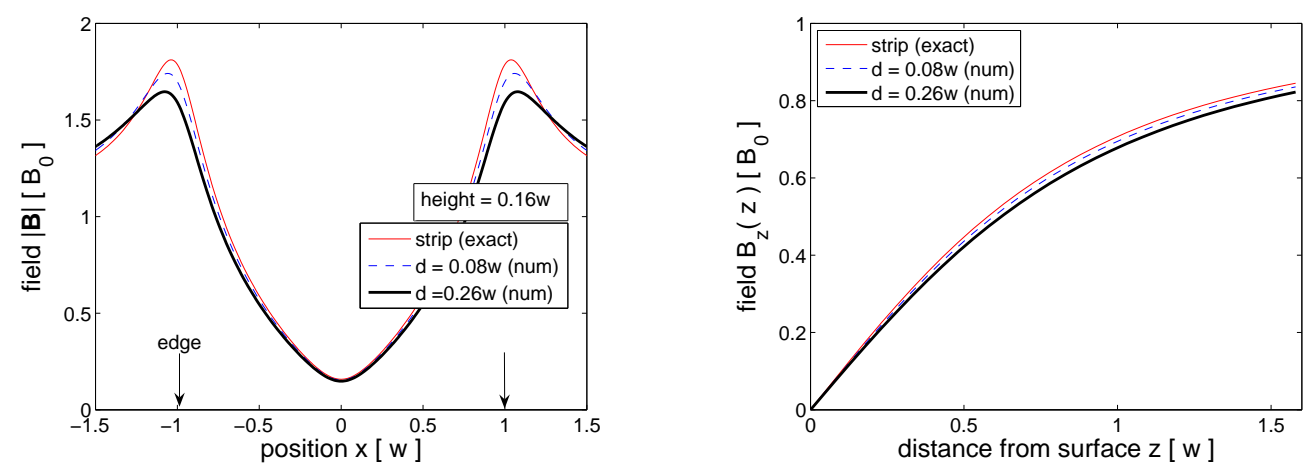

Figure 4: Modulus of magnetic field above a thin superconducting strip placed in a vertical magnetic field of magnitude $B_{0}$. (left) Field at fixed height, $|\mathbf{B}(x, z=0.16 w)|$, given in the units of the applied field $B_{0}$. (right) Field $|\mathbf{B}(x=0, z)|$. The position $x=0$ corresponds to the strip centre above which the field is oriented perpendicular to the strip; due to symmetry and continuity, it must go to zero at the wire surface. The lines compare calculations for strips of different thickness $d$. Infinitely thin strip: analytical calculation based on Ref.[35]; $d>0$ : numerical calculations.

strip, the distribution of the sheet current is given by the expression [35]:

$$
J\left(x, J_{c}, I\right)= \begin{cases}\left(2 J_{c} / \pi\right) \arctan \sqrt{\frac{w^{2}-b^{2}}{b^{2}-x^{2}}}, & |x|<b \\ J_{c}, & b<|x|<w\end{cases}
$$

where $b=w\left(1-I^{2} / I_{c}^{2}\right)^{1 / 2}, I_{c}=2 w J_{c}$ is the maximal value for a superconducting current in the strip (critical current); it is achieved at full magnetic field penetration, $b=0$. The distribution of the sheet current is presented in Fig [5 (top left). Eq.(4) applies when the transport current $I$ has been increased from zero (virgin state) for the first time (cyclic current change is discussed below).

Due to symmetry, the side guide is located above the strip centre when the bias field is parallel to the $x$-axis. Fig.5 (bottom left) presents the magnetic field produced by a current $I$ on the axis $x=0$ of an infinitely thin strip of width $2 w$ in the mixed state. The dependence of the magnetic field on the distance $z$ was calculated numerically for four ratios of the current to the critical current $I / I_{c}$ (the same as in Fig 5 (top left)). The data should be compared to Fig.3 where a superconductor in the Meissner state and a normal conductor are considered. The magnetic trapping potential that is formed in combination with a horizontal bias field (of magnitude $2.5 \mu_{0} I /\left(2 \pi^{2} w\right)$ ), are shown in Fig 5 (bottom right). These plots demonstrate the tendency: the closer the current to the critical current, the closer are the trap parameters to the values of a normal metal trap. 

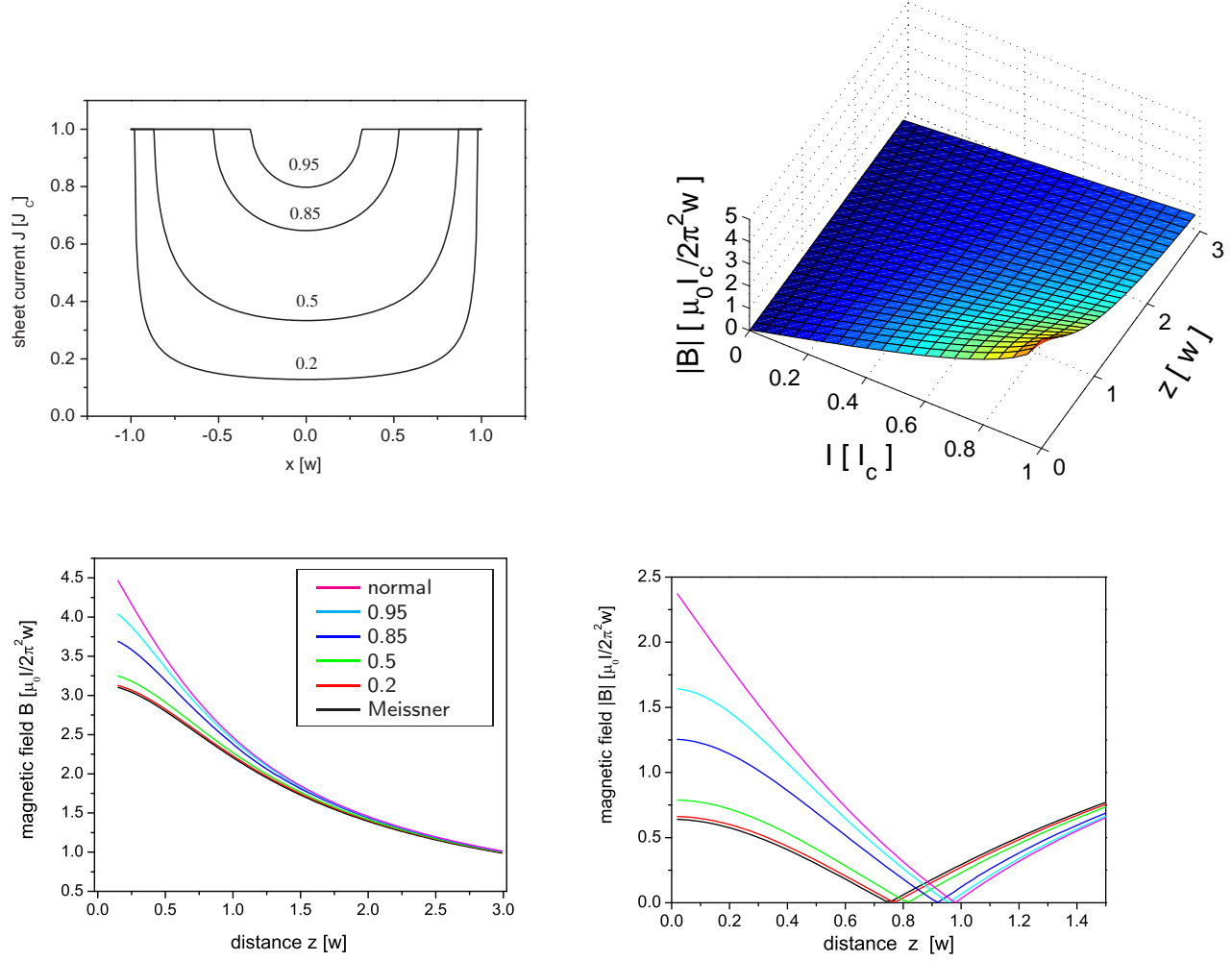

Figure 5: (top left) Sheet current distribution in a thin strip of type-II superconductor as a function of the transport current $I$ which is increased from zero (virgin state). The curves are plotted for $I / I_{c}=0.2,0.5,0.85$, and 0.95 where $I_{c}=2 d w j_{c}$ is the (total) critical current; (top right) Magnetic field vs. distance and current above the centre of the strip, $x=0$, with no external (bias) field. For a critical current density of $j_{c}=10^{11} \mathrm{~A} / \mathrm{m}^{2}$ the field reaches a maximum value of $\mu_{0} I_{c} /\left(2 \pi^{2} w\right) \approx 127 \mathrm{G}(d / 1 \mu \mathrm{m})$ at $I=I_{c}$ and at the wire surface. (bottom left) Magnetic fields calculated as a function of distance for different $I / I_{c}$ ratios, as given in the legend: black - Meissner state; red ... cyan $I / I_{c}=0.2 \ldots 0.95$; magneta - normal metal. The field is normalized to $\mu_{0} I /\left(2 \pi^{2} w\right)$, as in Fig.2. The calculations were made numerically using the current distributions given by (4). (bottom right) The "mixed-state" magnetic potentials as a function of distance $z$ at $x=0$ are presented for the same $I / I_{c}$ ratios. The horizontal bias field equals $2.5 \mu_{0} I /\left(2 \pi^{2} w\right)$. 
It should be noted that a superconductor in the mixed state is essentially a nonlinear system. As can be seen from the 3D-plot presented in Fig 5 (top right) the magnetic field is a nonlinear function of the current, in contrast to both a normal conducting wire and a superconducting one in the Meissner state. This happens because the shape of the current distribution depends on the ratio $I / I_{c}$ [see Fig $[5$ (top left)]. The calculations show that for low currents $\left(I<0.2 I_{c}\right)$, the nonlinearity is negligible and the field distribution around the type II superconducting strip may be described by the expressions obtained for a strip in the Meissner state in Section 3.1. In the opposite case, $I \cong I_{c}$, the current density equals the critical value over almost the whole strip width. The magnetic field around the strip can then be calculated as for a normal metal, with a spatially uniform current density.

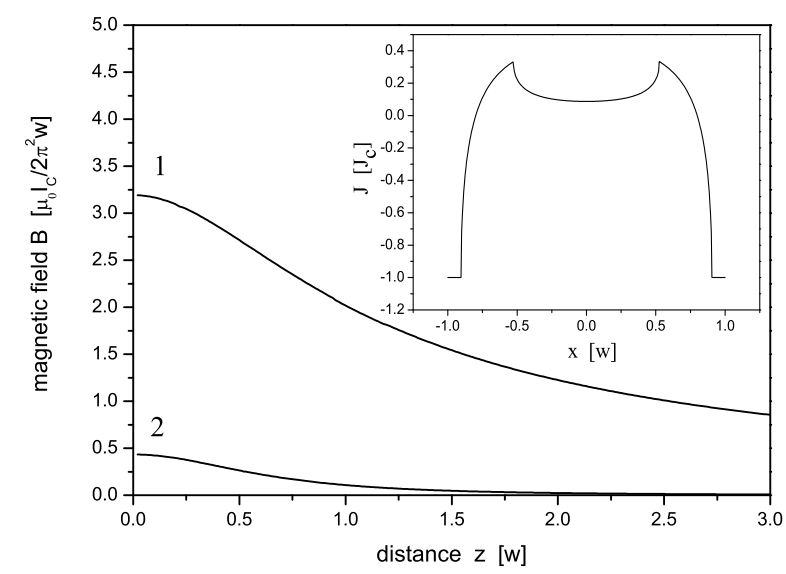

Figure 6: Illustration of trapped magnetic flux after ramping up and down a transport current in a type II superconducting wire in the mixed state. We plot the magnetic field produced by a transport current $I=0.85 I_{c}$ above the centre of a strip wire (curve 1), and the magnetic field caused by the flux trapped in the strip, after decreasing the current again to zero (curve 2). The magnetic field is given in units of $\mu_{0} I_{c} /\left(2 \pi^{2} w\right)$. The inset shows the remnant sheet current $J(x)$ in the strip after the current cycle, normalised to the critical sheet current $J_{c}$. The calculation follows Ref.[35].

\subsubsection{Trapped magnetic flux and related noise}

The capability of type II superconductors to freeze a magnetic flux results in the irreversible behavior of the current profile in the mixed state strip under cyclic 
changes of the external fields or transport currents. Fig 6 presents the magnetic field above the superconducting strip after ramping up the transport current to $I=0.85 I_{c}$ (curve 1) and decreasing it again to zero (curve 2). The inset shows the inhomogeneous current distribution that is left in the strip as a result of the freezing of the magnetic flux in this current cycle: in the centre, the current keeps flowing in the direction of the peak transport current, while near the edges it flows backwards. At short distance $(z<0.2 w)$ the frozen magnetic field is about $15 \%$ of the field produced by the maximum current. The ratio of these fields decreases approximately like $1 / z^{2}$ and is about $1 \%$ at $z=3 w$. These calculations were made for zero external (bias) field in the framework of the Brandt model [35].

The effect of the frozen magnetic field has to be taken into account for the proper loading and control of the magnetic trap. The frozen flux can also be the main source of magnetic noise generated by the guiding wire because of the motion of the trapped vortices. Other effects which can influence the atom cloud above mixed state wires are the variations of the critical current density due to local changes in the magnetic field or due to structure inhomogeneities. In addition to the vortex lattice, these effects could corrugate the trapping potential at low trap heights, $z_{t} \leq w$. Changes on slow time scales may occur due to the re-distribution of the frozen flux [40, 41].

The magnetic noise (flux noise) due to the motion of the trapped vortices is much higher than the noise in the Meissner state. The flux noise was investigated in many experimental [42, 43, 44] and theoretical [42, 44, 45] works. It was shown that it is closely related to the mechanism of vortex pinning which in turn depends on material properties and preparation technology of the sample. The flux noise intensity is determined by external conditions as well. When the temperature, current and magnetic field are far from the critical values $T_{c}, J_{c}$, and $B_{c 2}$, the noise arises from thermally activated, mutually incoherent hoppings among pinning centres. For this regime of "flux creep", SQUID-measurements near high- $T_{c}$ films demonstrate that the noise is strongly dependent on the film quality [43]. In addition, the flux noise picture is more complicated: the noise level depends on the magnetic pre-history, it follows not only the number of trapped vortices, but also the specific spectrum of metastable trapped states. In particular, a long-lived noisy state may occur in a superconducting wire after a pulsed transport current if the current is not small compared to the critical value [43, 48].

The influence of the magnetic pre-history is obviously harmful for controlling cold atom traps near a superconducting surface. Further to the additional flux noise, the vortices trapped in the superconductor produce magnetic disorder 
because of the arbitrary locations of the pinning centres. Note that cooling of the wire through the superconducting transition in an external magnetic field may also lead to the freezing of vortices. SQUID studies of the remnant noise due to vortices trapped in a weak field show that the flux noise spectral density at low frequencies decreases linearly, as the magnetic field in which the superconducting transition occurred, is lowered [43, 49]. In fact, the remnant noise is proportional to the number of vortices trapped in the sample in agreement with the Dutta, Dimon and Horn model [47]. This number is also determined by the shape of the superconductor: the narrower the strip, the less vortices are frozen. As was shown in Ref.[50], a niobium strip of width $2 w=10 \mu \mathrm{m}$ placed in a vertical magnetic field smaller than $\approx 0.5 \mathrm{G}$ does not freeze vortices while being cooled through $T_{c}$. At the same time, for a width of $100 \mu \mathrm{m}$, the critical field for vortex freezing is less than $4 \mathrm{mG}$ [50]. These observations indicate the advantageous nature of narrow superconducting wires for atom chip experiments which require particularly long lifetimes and coherence times.

To summarise the possibility of using mixed state wires for atom guiding and trapping, the advantage over the Meissner state is the higher current and thus the tighter confinement, while the disadvantage is the expected static potential corrugation and higher noise. It may even turn out that their performances are comparable to other, normally conducting materials that have been suggested recently [51, 52], but more detailed investigations are needed here. On the other hand, it may be possible to trap cold atoms close enough and long enough so that they may be considered as a probe of the mixed state of type II superconductors. The low-frequency flux noise in the mixed state could be detected by the spin dephasing rate of cold atoms, as was noted in [53]. The high sensitivity of cold atoms to a disturbance of the magnetic potential could be used for visualization of the static disorder produced by frozen vortices, analogously to the current static scattering in normal atom chip wires [54, 18, 19]. Atom clouds having high spatial resolution $(3 \mu \mathrm{m})$ combined with excellent sensitivity to magnetic field (4 nT) [54] (or even better than that [55]) could provide complementary information about the distribution and dynamics of vortices. According to the review in Ref.[56], such a combination of resolution and sensitivity would be one of the best among various vortex observation methods. 


\section{Comparison between normal and superconduct- ing magnetic traps}

Our calculations show that magnetic traps can be created in atom chips with superconducting wires. The main differences to normal wires are the inhomogeneity of the current distribution and nonzero screening currents induced by bias fields. The current density in a superconducting wire is smaller in its centre compared to a normal conductor (at the same total current). For this reason, the magnetic field near the wire surface is weaker, and a side guide trap (in a given parallel bias field) is closer to the surface (see Fig 3 ).

Let us now analyse in more detail the difference in trap parameters between side-guide traps created by the normal and superconducting guiding wires. We also compare the Meissner and mixed states with respect to their trapping "capabilities". Note that we do not take into account here the bending of the wires into "U"- and "Z"-shapes for 3D traps (see, for example, [1, 2, 3]). Calculation of the current density in bent superconducting wires is complicated because it requires to solve a three-dimensional problem even in a planar configuration [20]. Our results can be applied to the central part of the wires, sufficiently far away from the bends.

Two figures of merit describing the confinement of cold atoms in the magnetic trap are used for this comparison: the magnetic field gradient at the trap centre and the depth of the trapping potential. The trap depth is determined as the minimal height of the (total) potential barrier, from the trap centre to either the surface or away from it. Here, the gravitational potential is taken into account. We adjust the trap height by setting the bias field to the required value and take the same geometry and total current for a fair comparison between superconducting and metallic wires.

Results for typical flat wires are shown in Fig.7. Comparing superconducting and normal guiding wires, we see that the field gradients (left scale) and the trap depths are essentially the same for trap distances $z_{t} \geq 2 w$, similar to what is seen in Fig. The difference is maximal in the range of low trap heights, where one becomes sensitive to the weaker magnetic field and current density in the superconductor. This result is in qualitative agreement with the numerical simulations of confinement parameters of specific superconducting traps performed in [20]. The finite thickness of the wire does not significantly change the picture, as can be seen from the numerical data plotted in Fig.7 (b) (symbols). For example, an infinitely thin superconducting strip creates a magnetic field gradient along the 


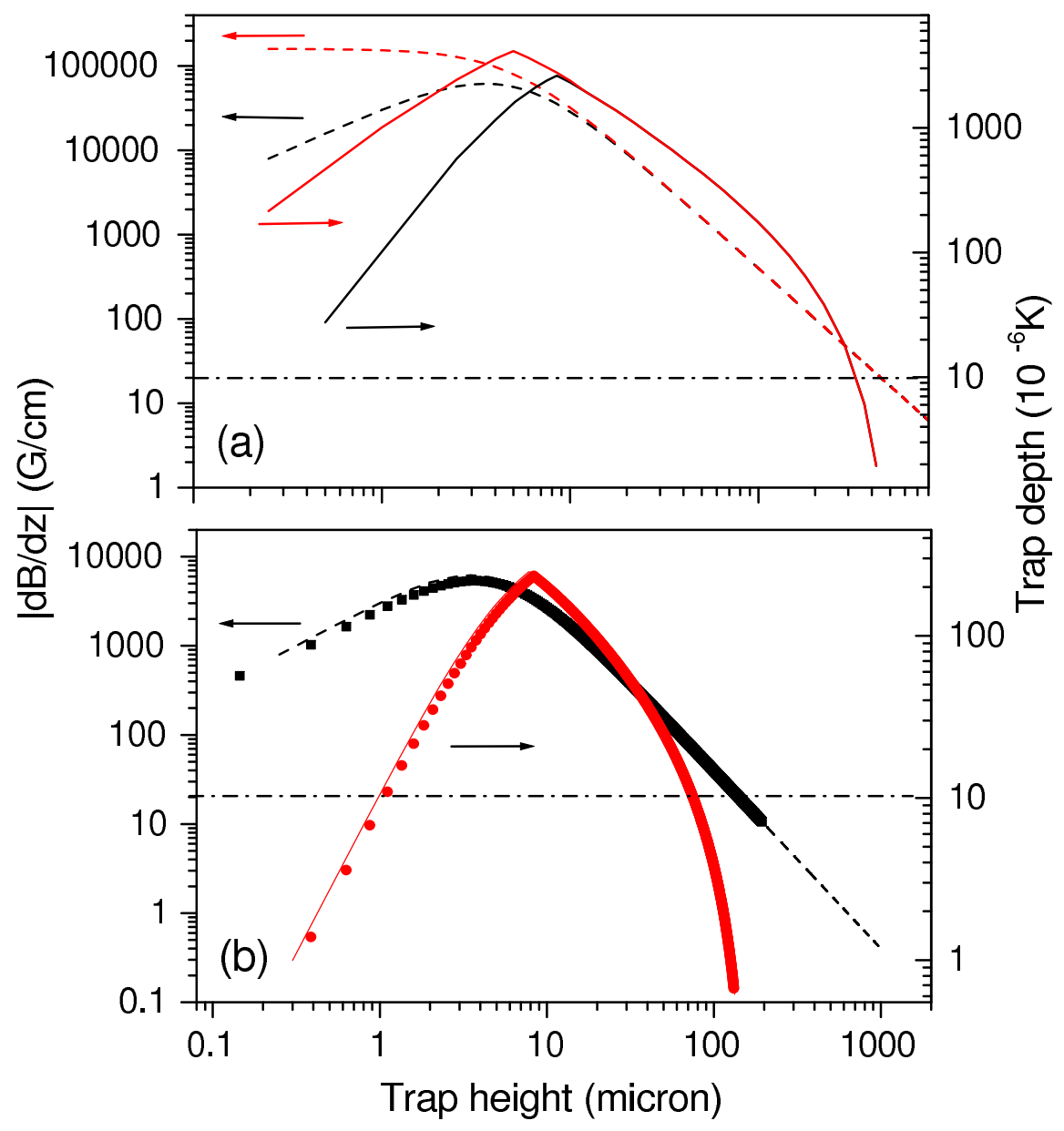

Figure 7: Comparison of trap parameters (field gradient and trap depth) calculated for side-guide traps with superconducting and normal metal wires. (a) Superconductor in the mixed state $\left(\mathrm{YBa}_{2} \mathrm{Cu}_{3} \mathrm{O}_{7-\delta}\right.$ at $\left.4.2 \mathrm{~K}\right)$ and normal metal (black and red lines, respectively). Calculations were performed analytically for infinitely thin guiding wires of width $2 w=10 \mu \mathrm{m}$, and current $I=200 \mathrm{~mA} \approx 0.1 I_{c}$ (assuming a thickness $d=300 \mathrm{~nm}$ ). The trap height is varied by adjusting the bias field. Dashed lines: absolute value of magnetic field gradient $|d B / d z|$; solid lines: trap depth. (b) Superconductor in the Meissner state (niobium at $4.2 \mathrm{~K}$ ). Lines: analytical calculation for an infinitely thin wire of width $2 w=10 \mu \mathrm{m}$, carrying $20 \mathrm{~mA}$ of current. Black and red squares: numerically determined trap parameters for a strip with thickness $d=2 \mu \mathrm{m}$. The numerical calculation takes into account the additional screening currents created by the bias field (negligible for an infinitely thin wire). Dash-dotted (horizontal) lines: minimum field gradient required to stabilize the trap against gravity and minimum trap depth to confine a cold atomic sample at $1 \mu \mathrm{K}$. The trap depth is calculated from the height of the potential barriers towards or away from the chip, including gravity ("upside down" setup with the trap below the chip). 
$z$-direction at the trap centre (height $z_{t}=0.75 w$ ) that is about 1.8 times less than in a normal metal trap. Along the $x$-axis the ratio of the gradients (normal metal to superconductor) is about 1.3.

The data presented in Fig.7 are calculated for the high- $T_{c}$ superconductor $\mathrm{YBa}_{2} \mathrm{Cu}_{3} \mathrm{O}_{7-\delta}(\mathrm{YBCO})$ and for niobium $(\mathrm{Nb})$ in the Meissner state. If the YBCO film is used at $4.2 \mathrm{~K}$, the assumed current of $200 \mathrm{~mA}$ is about $10 \%$ of the critical value (critical current density $j_{c} \approx 7 \cdot 10^{11} \mathrm{~A} / \mathrm{m}^{2}$ ). Nevertheless, we expect the wire to be in the mixed state, at least at its edges where the current density exceeds the values permitting the Meissner state. For niobium, the critical current density is lower, and this is why we reduce the transport current to $20 \mathrm{~mA}$. The horizontal lines in Fig.7 mark typical criteria for reliable trapping of atoms at a temperature of $1 \mu \mathrm{K}$ : the trap depth should exceed $10 \mu \mathrm{K}$ and the gradient should be high enough to protect the atoms from gravity's pull (corresponding to $15.3 \mathrm{G} / \mathrm{cm}$ for ${ }^{87} \mathrm{Rb}$ atoms in the $\left|F=2, m_{F}=2\right\rangle$ state, where $F$ is the total spin and $m_{F}$ its projection on the local magnetic field).

According to Fig.7(a), we predict that an atom chip based on a YBCO superconducting strip can trap cold atoms in a wide range of trap heights $0.2 \div 300 \mu \mathrm{m}$. For niobium, the range is smaller $(1 \div 75 \mu \mathrm{m})$ due to the lower guiding current (Fig:7(b)). The trap parameters are still high enough, however, to successfully trap cold atoms, both in the Bose-Einstein condensed phase and above. It is also seen in Fig 7 that the trap parameters in the closest vicinity of the surface $(z \leq 5 \mu \mathrm{m})$ are worse than for a normal strip. However, a significant gain in the lifetime due to the reduction of magnetic noise near the superconductor makes this trap design more attractive.

\section{Conclusion}

We have presented a theoretical analysis of superconducting atom chips. Our methods have been both analytical and numerical; they complement the results recently reported in Ref.[20]. In particular, the analytical expressions given here can be used for semi-quantitative estimates and to identify scaling laws for chip design. We confirm the possibility of trapping cold atoms in a wide range of distances $(0.2 \div 300 \mu \mathrm{m})$ with the same wire. This analysis takes into account the specific behavior of superconductors that carry a transport current in a magnetic field. These peculiarities enforce modifications in the loading procedure and the control of the atom cloud. The application of superconductors to atom chips may 
enable improved atom optics with suppressed effects of noise, as well as novel insight regarding the noise and current distribution in superconductors.

\section{Acknowledgments}

We acknowledge the support of the Marie-Curie programme of the European Union, from the Bundesministerium für Bildung und Forschung (Germany, DIP project), the German-Israeli Foundation for Scientific Research (GIF), the American-Israeli Foundation (BSF), and the Israeli Science Foundation. C.H. thanks the Deutsche Forschungsgemeinschaft (DFG) for support (He 2849/3).

\section{A Side guide magnetic trap based on cylindrical su- perconducting wire}

Let us consider a conductor in the form of a cylinder (radius $R$ ) with a DC transport current $I$ in an external magnetic field $\mathbf{B}_{0}$ (Fig 8). A normal metal wire does not influence the external DC field so that the total field is the superposition of the homogeneous bias field and the field produced by the current. The total field is zero at $\theta=0$ and at the trap height

$$
z_{t}=r_{t}-R=\frac{\mu_{0} I}{2 \pi B_{0}}-R
$$

A magnetic field cannot penetrate a superconductor in the Meissner state. If the radius is much larger than the London penetration depth, the magnetic field is zero inside the cylinder. The magnetic field around the superconductor without transport current can be described by the Laplace equation for the scalar magnetic potential $\psi$. The boundary conditions are: the radial component of the magnetic field equals zero at $r=R$; at $r \rightarrow \infty$, the magnetic field coincides with the external one. The solution is

$$
\begin{aligned}
& B_{r}=-\partial_{r} \psi=B_{0}\left(1-\frac{R^{2}}{r^{2}}\right) \sin \theta \\
& B_{\theta}=-\frac{1}{r} \partial_{\theta} \psi=B_{0}\left(1+\frac{R^{2}}{r^{2}}\right) \cos \theta
\end{aligned}
$$

where $B_{r}$ and $B_{\theta}$ are the radial and azimuthal components of the magnetic field. The magnetic field configuration around the superconductor thus differs from a 


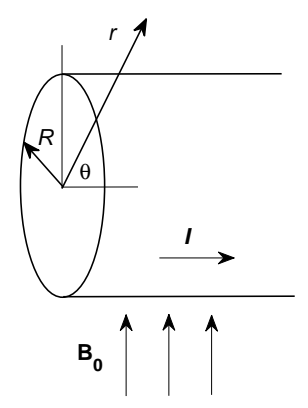

Figure 8: Cylindrical wire and coordinate system.

normal metal even if a transport current is absent. Due to the Meissner effect, the magnetic field near the superconductor at $\theta=0$ is increased.

If the cylinder carries a transport current, the outer field is the same as for a normal conductor, due to the cylindrical symmetry. We find that the minimum of the total field (Fig. 9 (left)) is located at $\theta=0$ and distance

$$
z_{t}=r_{t}-R=R \frac{1+\sqrt{1-4 h^{2}}}{2 h}-R, \quad h=\frac{2 \pi R B_{0}}{\mu_{0} I}
$$

from the wire surface. Because of the screening of the bias field by a superconductor, the same trap height in a superconducting chip is achieved with a lower bias field than in a normal conducting chip (see Fig 9 (right) and also Fig 3 (right) for a rectangular wire). This reduction reaches $50 \%$ at small trap heights. The difference decreases with an increase of this height and practically vanishes at $z_{t}>3 R$.

\section{B Numerical calculation of magnetic field around a finite thickness superconductor}

We consider a wire that is infinitely long in the $y$-direction and ignore here boundary effects (these become relevant for " $U$ "- and "Z"-shaped wires, of course). By symmetry, the magnetic field is independent of $y$ and lies in the $x z$-plane (see 

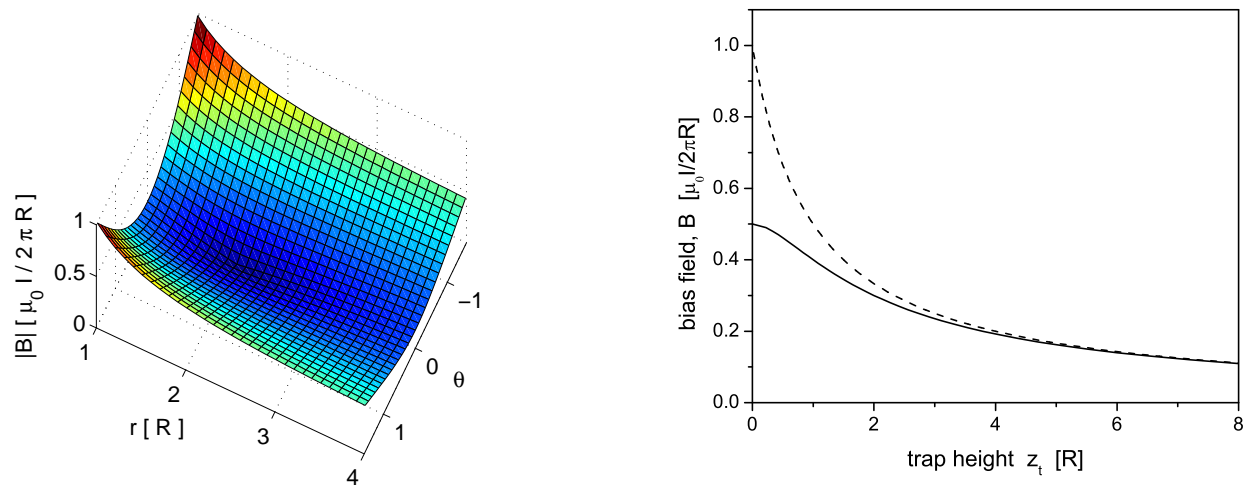

Figure 9: (left) Modulus of the total magnetic field around a superconducting cylinder with a transport current $I$ in a bias magnetic field $B_{0}=0.4 \times \mu_{0} I / 2 \pi R$. The field minimum is located at the point $\theta=0, z_{t}=0.8 R(r=1.8 R)$. (right) Required bias field as a function of the trap height for a cylindrical wire, based on Eqs.(5) and (8): solid line - superconductor; dashed line - normal metal. In both graphs, the magnetic field is normalised to $\mu_{0} I / 2 \pi R=2 \mathrm{G}(I / 1 \mathrm{~A}) /(R / 1 \mathrm{~mm})$.

Fig.1). Inside the superconductor, the field is zero. Outside, we describe the field in terms of a scalar potential $\psi$ and a vector potential $\mathbf{A}=\mathbf{e}_{y} A$

$$
\mathbf{B}(\mathbf{x})=-\nabla \psi(\mathbf{x})+\nabla \times \mathbf{A}(\mathbf{x}) .
$$

We shall see below that the scalar potential describes the field caused by an external magnetic field (as in Appendix A), while the vector potential gives the field caused by a transport current in the superconducting wire. The introduction of two potentials may seem superfluous, because one could work, outside the wire, with the scalar potential only. But $\psi$ would then become a multivalued function for a nonzero transport current.

The magnetic field normal to the superconductor surface is continuous and must therefore vanish. (This corresponds to the radial derivative of $\psi$ in Appendix A.) Writing $\mathbf{n}\left(\mathbf{x}_{s}\right)$ for the normal at surface point $\mathbf{x}_{s}$, we have

$$
\begin{aligned}
\mathbf{n}\left(\mathbf{x}_{s}\right) \cdot \mathbf{B}\left(\mathbf{x}_{s}\right) & =-\mathbf{n}\left(\mathbf{x}_{s}\right) \cdot \nabla \psi\left(\mathbf{x}_{s}\right)-\left(\mathbf{n}\left(\mathbf{x}_{s}\right) \times \mathbf{e}_{y}\right) \cdot \nabla A\left(\mathbf{x}_{s}\right) \\
& =-\frac{\partial \psi\left(\mathbf{x}_{s}\right)}{\partial n\left(\mathbf{x}_{s}\right)}-\frac{\partial A\left(\mathbf{x}_{s}\right)}{\partial t\left(\mathbf{x}_{s}\right)}=0
\end{aligned}
$$

where $\partial / \partial n\left(\mathbf{x}_{s}\right)$ and $\partial / \partial t\left(\mathbf{x}_{s}\right)$ are the normal and tangential derivatives at $\mathbf{x}_{s}$. (The local tangent vector is $\mathbf{t}=\mathbf{n} \times \mathbf{e}_{y}$.). We construct the potentials $\psi$ and $A$ such 
that both terms $\partial \psi_{s} / \partial n$ and $\partial A_{s} / \partial t$ in Eq. (10) are zero. Note that $\partial A_{s} / \partial t=0$ indicates that $A\left(\mathbf{x}_{s}\right)$ is a constant on the surface which we denote by $A_{0}$. The tangential magnetic field is nonzero at the (outer) surface of the superconductor; the interior of the wire is screened from this field by a surface current (along the $y$-axis) of magnitude $\mathbf{t} \cdot \mathbf{B} / \mu_{0}$.

Considering that outside the wire, both divergence and curl of the magnetic field are zero, we get

$$
\nabla^{2} \psi(\mathbf{r})=0, \quad \nabla^{2} A(\mathbf{r})=0
$$

where $\nabla^{2}$ is the two-dimensional Laplace operator. These Laplace equations can be solved with the help of the Green function

$$
G(\mathbf{x}, \mathbf{r})=-\frac{1}{2 \pi} \log r
$$

where $r=|\mathbf{r}-\mathbf{x}|$, given the values of the potentials and their derivatives on the wire surface, and an asymptotic condition at large distance.

The two potentials behave differently with respect to the current $I$ transported by the superconductor. It can be seen by recalling the Ampère-Maxwell law (in the static limit)

$$
\mu_{0} I=\oint_{S} \mathbf{B}(\mathbf{x}) \cdot \mathrm{d} \mathbf{a}=-\oint_{S} \frac{\partial A}{\partial n} \mathrm{~d} a,
$$

where $I$ is the total current flowing through the superconductor, $S$ a closed curve including the cross-section of the superconductor (with oriented line element da), and $\partial / \partial n$ is the derivative normal to the curve $S$ and pointing 'outside'. Hence, the vector potential $A$ is proportional to the transport current. Conversely, the scalar potential asymptotically goes over into $\psi \rightarrow \psi_{\text {ext }}(\mathbf{r})=-\mathbf{r} \cdot \mathbf{B}_{0}$ where $\mathbf{B}_{0}$ is the homogeneous bias field. This asymptotic condition forces $\psi$ to be proportional to $\mathbf{B}_{0}$.

We solve the Laplace equations (11) for the potentials $M=\psi, A$ in terms of a surface integral equation ( $r$ outside the wire) [57, 58]

$$
M(\mathbf{r})=M_{\mathrm{ext}}(\mathbf{r})-\oint_{S} \mathrm{~d} a(\mathbf{x})\left(G(\mathbf{x}, \mathbf{r}) \frac{\partial M(\mathbf{x})}{\partial n(\mathbf{x})}-\frac{\partial G(\mathbf{x}, \mathbf{r})}{\partial n(\mathbf{x})} M(\mathbf{x})\right)
$$

where $M_{\text {ext }}$ is the external potential (nonzero only for $\psi$ ), $S$ is now the circumference of the wire (with scalar line element $\mathrm{d} a(\mathbf{x})$ ), and the normal derivative $\partial / \partial n(\mathbf{x})$ points 'outside' the wire. For $M=\psi$, we have $\partial \psi(\mathbf{x}) / \partial n(\mathbf{x})=0$ on the surface according to Eq.(10); then there is only one unknown, $\psi(\mathbf{x})$, in Eq.(14). 
When $M=A$, we can set under the integral $A(\mathbf{x})=A_{0}$, constant on the surface, also according to Eq.(10). Then the integral of the second term in the bracket can be shown to vanish, and there is only one unknown left, $\partial A(\mathbf{x}) / \partial n(\mathbf{x})$, which is actually the surface current density, see Eq.(13). We always choose $A_{0}=1$ in the beginning, compute the total current by Eq.(13) (giving a coefficient like the wire inductance) and re-scale $A(\mathbf{x})$ and $\partial A / \partial n(\mathbf{x})$ to get the desired current.

The potential $M$ and its derivative on the surface are obtained by letting $\mathbf{r} \rightarrow$ $\mathbf{x}_{s}$ in Eq. (14) approach the surface. Note that we touch the singularity of the Green function $G\left(\mathbf{x}, \mathbf{x}_{s}\right)$ and its normal derivative $\partial G\left(\mathbf{x}, \mathbf{x}_{s}\right) / \partial n(\mathbf{x})$ under the integral when $\mathrm{x}=\mathrm{x}_{s}$. To handle this, we expand the integrand within a small neighborhood $s$ of length $\mathrm{d} a$ around $\mathbf{x}_{s}$ and perform the integration, giving

$$
\begin{gathered}
\int_{s} \mathrm{~d} a(\mathbf{x}) G\left(\mathbf{x}, \mathbf{x}_{s}\right) \frac{\partial M(\mathbf{x})}{\partial n(\mathbf{x})} \approx \frac{1}{2 \pi} \frac{\partial M\left(\mathbf{x}_{s}\right)}{\partial n\left(\mathbf{x}_{s}\right)}\left(1-\log \frac{\mathrm{d} a}{2}\right) \mathrm{d} a . \\
\int_{s} \mathrm{~d} a(\mathbf{x}) \frac{\partial G\left(\mathbf{x}_{s}, \mathbf{x}\right)}{\partial n(\mathbf{x})} M(\mathbf{x}) \approx\left(\frac{1}{2}+\frac{\mathrm{d} \phi\left(\mathbf{x}_{s}\right)}{4 \pi}\right) M\left(\mathbf{x}_{s}\right) .
\end{gathered}
$$

Here the angle $\mathrm{d} \phi\left(\mathbf{x}_{s}\right)$ involves the radius of curvature $R\left(\mathbf{x}_{s}\right)$ of the surface: $\mathrm{d} \phi\left(\mathbf{x}_{s}\right)=\mathrm{d} a / R\left(\mathbf{x}_{s}\right)$, it describes the angle subtended by the surface element as seen from the centre of curvature. The logarithmic correction of Eq. (15) and the curvature correction (16) greatly improve the convergence of the numerical calculations.

The other parts of the surface integral, excluding the point $\mathbf{x}_{s}$, are discretized in the usual way, mapping the integral equation into a linear system [59, 60]. For example, a rectangle $20.64 \mu \mathrm{m} \times 0.84 \mu \mathrm{m}$ with rounded corners $(R=0.32 \mu \mathrm{m})$ is typically discretized into 420 surface elements along its circumference. This leads to a linear system with $420^{2}$ matrix elements. Once we have the potential or its derivative on the wire surface, the field outside the wire is found from Eq. (14). We have checked the convergence of the numerics, for different discretizations of the wire surface, against the exact solution of Appendix A for cylindrical wires and against the solution of Ref.[61] for rectangular wires with sharp corners.

\section{References}

[1] R. Folman, P. Krüger, J. Schmiedmayer, J. Denschlag, and C. Henkel, Adv. At. Mol. Opt. Phys. 48, 263 (2002).

[2] J. Reichel, Appl. Phys. B 74, 469 (2002). 
[3] J. Fortágh and C. Zimmermann, Rev. Mod. Phys. 79, 235 (2007).

[4] T. Varpula and T. Poutanen, J. Appl. Phys. 55, 4015 (1984).

[5] C. Henkel, S. Pötting, and M. Wilkens, Appl. Phys. B 69, 379 (1999).

[6] J. A. Sidles, J. L. Garbini, W. M. Dougherty, S. H. Chao, Proc. IEEE 91, 799 (2003); quant-ph/0004106.

[7] S. Scheel, P. K. Rekdal, P. L. Knight and E. A. Hinds, Phys. Rev. A 72, 042901 (2005).

[8] D. M. Harber, J. M. McGuirck, J. M. Obrecht, and E. A. Cornell, J. Low. Temp. Phys. 133, 229 (2003); condmat/0307546.

[9] Y. Lin, I. Teper, C. Chin, and V. Vuletic, Phys. Rev. Lett. 92, 050404 (2004).

[10] M. P. A. Jones, C. J. Vale, D. Sahagun, B. V. Hall, and E. A. Hinds, Phys. Rev. Lett. 91, 080401 (2003).

[11] B. K. Skagerstam, U. Hohenester, A. Eiguren, and P. K. Rekdal, Phys. Rev. Lett. 97, 070401 (2006).

[12] P. K. Rekdal and B. K. Skagerstam, Phys. Rev. A 75, 022904 (2007).

[13] B. K. Skagerstam, and P. K. Rekdal, Phys. Rev. A 76, 052901 (2007).

[14] U. Hohenester, A. Eiguren, S. Scheel,and E. A. Hinds, Phys. Rev. A 76, 033618 (2007).

[15] T. Nirrengarten, A. Qarry, C. Roux, A. Emmert, G. Nogues, M. Bruno, J.M. Raimond, and S. Haroche, Phys. Rev. Lett. 97, 200405 (2006); C. Roux, A. Emmert, A. Lupascu, T. Nirrengarten, G. Nogues, M. Brune, J.-M. Raimond, and S. Haroche, Europhys. Lett. 81, 56004 (2008).

[16] T. Mukai, C. Hufnagel, A. Kasper, T. Meno, A. Tsukado, K. Semba, and F. Shimizu, Phys. Rev. Lett. 98, 260407 (2007).

[17] B. Zhang, C. Henkel, E. Haller, S. Wildermuth, S. Hofferberth, P. Krüger, and J. Schmiedmayer, Eur. Phys. J. D, 35, 97 (2005).

[18] S. Aigner, L. Della Pietra, Y. Japha, O. Entin-Wohlman, T. David, R. Salem, R. Folman, and J. Schmiedmayer, Science 319, 1226 (2208). 
[19] Y. Japha, O. Entin-Wohlman, T. David, R. Salem, S. Aigner, J. Schmiedmayer and R. Folman, Phys. Rev. B 77, 201407(R) (2008).

[20] D. Cano, B. Kasch, H. Hattermann, D. Koelle, R. Kleiner, C. Zimmermann, and J. Fortágh, Phys. Rev. A 77, 063408 (2008).

[21] D. Saint-James, G. Sarma and E. J. Thomas, Type-II Superconductivity, Pergamon Press, 1969.

[22] D. K. Finnemore, T. F. Stromberg, and C. A. Swenson, Phys. Rev. 149, 231 (1966).

[23] Y. Takano, H. Takeya, H. Fujii, H. Kumakura, T. Hatano, K. Togano, H. Kito and H. Ihara., Appl. Phys. Lett. 78, 2914 (2001).

[24] S. Ghamati, H. Suhl, W. Vernon and G. Webb, IEEE Trans. Magn. 21, 831 (1985).

[25] Y. Yeshurun, A. P. Malozemoff, F. Holtzberg and T. R. Dinger, Phys. Rev. B 38, 11828 (1988).

[26] I. Matsubara, R. Funahashi, K. Ueno, H. Yamashita, T. Kawai, Physica C: Supercond. 256, 33 (1996).

[27] I. A. Rudnev, S. V. Antonenko, D. V. Shantsev, T. H. Johansen, Cryogenics 43, 663 (2003).

[28] R.L. Meng, T.H. Johansen, I.A. Rusakov, A. Baikalov, D. Pham, F. Chen, Z.Y. Zuo and C.W. Chu, Physica C: Supercond. 434, 39 (2006).

[29] D. Larbalestier, A. Gurevich, M. Feldmann, and A. Polyanskii, Nature 414, 368 (2001).

[30] S. Y. Xu, Qi Li, E. Wertz, Y. F. Hu, A. V. Pogrebnyakov, X. H. Zeng, X. X. Xi, and J. M. Redwing, Phys. Rev. B 68, 224501 (2003)

[31] Physical Properties of High Temperature Superconductors, edited by D. M. Ginsberg (World Scientific, Singapore, 1989), Vol. I.

[32] G. Blatter, M. V. Feigelman, A. I. Larkin, and V. M. Vinokur, Rev. Mod. Phys. 66, 1125 (1994). 
[33] "Physics and Materials Science of Vortex States, Flux Pinning and Dynamics", eds. R. Kossowsky, S. Bose, V. Pan and Z. Durusoy, NATO Sciences Series E: Applied Sciences - Vol. 356, Kluwer Academic Publishers, Dordrecht 1999.

[34] P. W. Anderson, Phys. Rev. Lett. 9, 309 (1962); P. W. Anderson, Y. B. Kim, Rev. Mod. Phys. 36, 39 (1964).

[35] E. H. Brandt and M. Indenbom, Phys. Rev. B 48, 12893 (1993); E. H. Brandt, Phys. Rev. B 46, 8628 (1992).

[36] E. Zeldov, J. R. Clem, M. McElfresh, and M. Darwin, Phys. Rev. B 49, 9802 (1994).

[37] E. M. Lifshitz, L. D. Landau, and L. P. Pitaevskii, "Electrodynamics of Continuous Media", Vol. 8 of Course of Theoretical Physics (ButterworthHeinemann, 1984).

[38] A. V. Kuznetsov, D. V. Eremenko, and V. N. Trofimov, Phys. Rev. B 59, 1507 (1999).

[39] C. P. Bean, Phys. Rev. Lett. 8, 250 (1962).

[40] A. V. Bobyl, D. V. Shantsev, Y. M. Galperin, T. H. Johansen, M. Baziljevich, and S. F. Karmarenko, Supercond. Sci. Technol. 15, 82 (2002).

[41] Y. Yeshurun, A. P. Malozemov, and A. Shaulov, Rev. Mod. Phys. 68, 911 (1996).

[42] J. R. Clem, Phys. Rep. 75, 1 (1981).

[43] M. F. Ferrari, M. Jonson, F. Wellstood, J. Clarke, D. Mittzi, P. A. Rosenthal, C. B. Eom, T. H. Geballe, A. Kaputilnik, and M. R. Beasley, Phys. Rev. Lett. 64, 72 (1990); M. F. Ferrari, M. Jonson, F. Wellstood, J. J. Kingston, T. J. Shaw and J. Clarke, J. Low Temp. Phys. 94, 15 (1994).

[44] B. Plaçais, P. Mathieu, and Y. Simon, Phys. Rev. B 49, 15813 (1994).

[45] V. D. Ashkenazy, G. Jung, B. Ya. Shapiro, Phys. Rev. B 51, 9118 (1995).

[46] E. H. Brandt, Rep. Prog. Phys. 58, 1465 (1995). 
[47] P. Dutta, P. Dimon, and P. M. Horn, Phys. Rev. Lett. 43, 646 (1979); P. Dutta and P. M. Horn, Rev. Mod. Phys. 53, 497 (1981).

[48] S. Kuriki, Y. Kawaguchi, K. Takahashi and M. Matsuda, J. Appl. Phys. 96, 2768 (2004).

[49] T. J. Shaw, J. Clarke, R. B. van Dover, L. F. Schneemeyer, and A. E. White, Phys. Rev. B 54, 15411 (1996);

[50] G. Stan, S. B. Field, and J. M. Martinis, Phys. Rev. Lett. 92, 097003 (2004).

[51] V. Dikovsky Y. Japha, C. Henkel, R. Folman, Eur. Phys. J. D, 35, 87 (2005).

[52] T. David, Y. Japha, V. Dikovsky, R. Salem, C. Henkel and R. Folman, Eur. Phys. J. D 48, 321 (2008).

[53] S. Scheel, R. Fermani, E. A. Hinds Phys. Rev. A 75, 064901 (2007).

[54] S. Wildermuth, S. Hofferberth, I. Lesanovsky, E. Haller, L. M. Andersson, S. Groth, I. Bar-Joseph, P. Krüger, and J. Schmiedmayer, Nature 435, 440 (2005); A. Günther, M. Kemmler, S. Kraft, C. J. Vale, C. Zimmermann, and J. Fortágh, Phys. Rev. A 71, 063619 (2005).

[55] M. Vengalattore, J.M. Higbie, S.R. Leslie, J. Guzman, L.E. Sadler, and D.M. Stamper-Kurn, Phys. Rev. Lett. 98, 200801 (2007).

[56] S. Oshima, K. Tanabe, T. Morishita, and M. Tonouchi, in: Vortex electronics and SQUIDS, edited by T. Kobayashi, H. Hayakawa and M. Tonouchi, Springer-Verlag Berlin Heidelberg, 2003.

[57] J.D. Jackson, Classical Electrodynamics, Wiley \& Sons, New York, 2nd edition, 1975.

[58] Manuel Nieto-Vesperinas, Scattering and diffraction in physical optics, John wiley \& Sons, 2nd edition, 1996.

[59] L. Rogobete and C. Henkel, Phys. Rev. A 70, 063815 (2004).

[60] Randy Bancroft, Understanding electromagnetic scattering using the Moment method: a practical approach, Artech House, Norwood, MA, 1996.

[61] E. H. Brandt and G. P. Mikitik, Phys. Rev. Lett. 85, 4164 (2000). 